\title{
Letter to the editor: Chlamydia trachomatis samples testing falsely negative in the Aptima Combo 2 test in Finland, 2019
}

Magnus Unemo', Damon Getman², Ronza Hadad ${ }^{1}$, Michelle Cole ${ }^{3}$, Nicholas Thomson ${ }^{4}$, Mirja Puolakkainen 5 , Gianfranco Spiteri6

1. WHO Collaborating Centre for Gonorrhoea and other STIs, National Reference Laboratory for STIs, Department of Laboratory Medicine, Faculty of Medicine and Health, Örebro University, Örebro, Sweden

2. Hologic Inc., San Diego, CA, United States

3. National Infection Service, Public Health England, London, United Kingdom

4. Wellcome Sanger Institute, Wellcome Genome Campus, Hinxton, United Kingdom

5. Department of Virology and Immunology, University of Helsinki and Helsinki University Hospital, Huslab, Helsinki, Finland

6. European Centre for Disease Prevention and Control (ECDC), Stockholm, Sweden

Correspondence: Magnus Unemo (magnus.unemo@regionorebrolan.se)

To the editor: We read with great interest the recent article by Rantakokko-Jalava et al. about false-negative Chlamydia trachomatis (CT) specimens in the nucleic acid amplification test (NAAT) Aptima Combo 2 assay (AC2; Hologic Inc.) [1]. These very important findings indicate that $6-10 \%$ of CT-positive cases in Finland might have been missed due to false-negative/equivocal $A C_{2}$ results. The $A C_{2}$ false-negative/equivocal $C T$ specimens were observed in several Finnish settings, particularly in south-western Finland, June 2018-April 2019. Finnish experts, together with Hologic scientists, quickly determined that the specimens with equivocal/ negative $\mathrm{CT}$ results in $\mathrm{AC} 2$ (target: $23 \mathrm{~S}$ rRNA) but positive in the Aptima Chlamydia trachomatis assay (ACT; target: 16S rRNA) were not linked to Hologic instruments or reagents. Concurrently, Finnish scientists identified a $\mathrm{C}_{1515} \mathrm{~T}$ mutation in the CT $23 \mathrm{~S}$ rRNA gene in $10 \mathrm{AC}_{2}$ false-negative specimens [1]. Further investigation and response is required and will need collaborative action.

Hologic has now provided strong evidence that the $\mathrm{C}_{1515} \mathrm{~T}$ mutation is the root cause of the $\mathrm{AC} 2$ falsenegative/equivocal CT specimens and confirmed in analytical studies that a synthetic RNA transcript corresponding to the CT $23 \mathrm{~S}$ rRNA with a $\mathrm{C}_{1515} \mathrm{~T}$ mutation yields significantly suppressed $C T$ detection probe-signal in $A_{2} 2$ similar to that observed with $A_{2}$ testing of clinical samples containing the mutated CT strain.

It is essential that a broader understanding is developed of the presence and proportion of specimens with mutated CT in Finland and internationally. Sufficient numbers of representative, geographically diverse false-negative $\mathrm{AC}_{2}$ specimens need to be examined in order to exclude the unlikely possibility of additional $23 \mathrm{~S}$ rRNA mutations affecting detection. Using $A C T$ for confirmation of $A C_{2}$ screened specimens provides clinical utility in the short term but is not a feasible long-term solution, because of the high cost and substantially increased work load and time for reporting results imposed by confirming a large number of $A C 2$ equivocal/negative specimens by $A C T$ testing. Additionally, the different performance characteristics of $A C 2$ and $A C T$ may further confound our understanding of the presence, spread and possible evolution of the new mutant (or, although unlikely, mutants). This might even result in the use of only ACT in some settings, which would create problems due to the widespread use of $\mathrm{AC}_{2}$ as well as loss of information regarding the presence of mutated specimens, presence of Neisseria gonorrhoeae, and the advantage of reserving $\mathrm{ACT}$ for confirmation testing.

Instead, until a revised version of the $\mathrm{AC} 2$ test is available, a validated and quality-assured 'mutant-specific' research-use-only test is urgently needed to understand the geographic distribution of the CT mutant and its prevalence in screening populations. To fulfil this need, Hologic is rapidly developing an Aptima-format research kit containing a $23 \mathrm{~S}$ rRNA-mutant detection probe. Surveillance using a test that detects the CT mutant will provide further timely insight into the impact of this CT variant on the effectiveness of CT screening in Finland and additional European countries where elevated levels of $\mathrm{AC}_{2}$ false-negative/equivocal results might be observed. In addition, this mutantspecific assay will generate evidence-based modifications to the current $A C 2$ assay to ensure accuracy of test results and patient safety. The European Centre for 
Disease Prevention and Control (ECDC) intends to facilitate such preferably centralised study or studies which will require close collaboration with Finland, additional European Union/European Economic Area countries, other international and national public health organisations, and Hologic.

The key public health priorities include understanding the spread of the CT mutant and ensuring that patients with false-negative results are recalled and tested using alternative NAAT. To address the former, any unexplained changes in $\mathrm{CT}$ epidemiology and positivity rate need to be further investigated. In laboratories using $A C_{2}$, retrospective and prospective review of the $\mathrm{AC} 2$ test results (proportions of positive, equivocal, negative test results, and associated relative light units (RLU) of test results) and investigations of any unexplained changes in the CT positivity rate should be performed. In settings with suspected false-negative CT test results by $\mathrm{AC}_{2}$, to ensure patient safety, specimens with low CT RLU values ( $\geq 15-99$ ), independent of the result interpretation in the Hologic instruments, need retesting with an appropriate NAAT targeting another genetic sequence (preferably ACT), and not by repeated $\mathrm{AC} 2$ testing. The look-back period for recalling patients with verified false-negative results will depend on the local proportion and epidemiology of the CT mutant; taking into account spontaneous clearance of CT infection, social consequences for the patients, potential risk of reinfection, and continuous analysis of positivity rates of retested patients.

Investigation of additional scientific issues will be important in due course, e.g. if it is only one mutated CT clone; the emergence (when, where and how), transmission and evolution of the clone(s) in different populations in Finland and possibly other countries; if the clone(s) has(ve) any fitness advantage/disadvantage; if only one or both the CT $23 \mathrm{~S}$ rRNA gene alleles are mutated in all specimens and how this affects the number or proportion of mutated $23 \mathrm{~S}$ rRNA molecules detected in $\mathrm{AC}_{2}$; if the lack of $\mathrm{AC}_{2}$ detection of the clone(s) has increased risk of CT-associated complications/sequelae; associations between the clone(s) and symptomatic/asymptomatic infection, and spread in different subpopulations (patient sex, age, sexual orientation, geographic distribution etc.). Whole genome sequencing from false-negative $\mathrm{AC} 2 \mathrm{CT}$ specimens is in progress to address several of these issues. Furthermore, a mutant-specific real-time PCR is also under development.

Finally, it is likely that mutants of CT and other infectious agents escaping detection in commercial and laboratory-developed diagnostic NAATs are more common than previously realised and are inevitable consequences of the microbial evolution combined with the high diagnostic selective pressure on the microorganisms. Several lessons on such diagnostically-selected evolution can be learnt from the identification of the Swedish new variant of CT (nvCT) in 2006 and its subsequent spread mainly in Sweden [2-5]. International and national surveillance programmes capturing diagnostic test escape mutants (as well as cross-reacting mutants of other pathogens) for $\mathrm{CT}$ and other pathogens should be considered, particularly for diagnostic NAATs with a single genetic target region.

\section{Acknowledgements}

We are grateful to Hologic Inc., San Diego, CA, United States for sharing unpublished scientific data.

\section{Conflict of interest}

DG is employed by Hologic Inc.; other authors: none declared.

Authors' contributions

MU wrote a first draft of the Letter; all authors commented and approved the final version of the Letter.

\section{References}

1. Rantakokko-Jalava K, Hokynar K, Hieta N, Keskitalo A, Jokela P, Muotiala A, et al. Chlamydia trachomatis samples testing falsely negative in the Aptima Combo 2 test in Finland, 2019. Euro Surveill. 2019;24(22):1900298. https:// doi.org/10.2807/1560-7917.ES.2019.24.22.1900298 PMID: 31164192

2. Ripa T, Nilsson P. A variant of Chlamydia trachomatis with deletion in cryptic plasmid: implications for use of PCR diagnostic tests. Euro Surveill. 2006;11(11):E061109.2. https:// doi.org/10.2807/esw.11.45.03076-en PMID: 17213548

3. Unemo M, Seth-Smith HM, Cutcliffe LT, Skilton RJ, Barlow D, Goulding D, et al. The Swedish new variant of Chlamydia trachomatis: genome sequence, morphology, cell tropism and phenotypic characterization. Microbiology. 2010;156(Pt 5):1394-404. https://doi.org/10.1099/mic.0.036830-0 PMID: 20093289

4. Herrmann B, Törner A, Low N, Klint M, Nilsson A, Velicko I, et al. Emergence and spread of Chlamydia trachomatis variant, Sweden. Emerg Infect Dis. 2008;14(9):1462-5. https://doi. org/10.3201/eid1409.080153 PMID: 18760021

5. Unemo M, Clarke IN. The Swedish new variant of Chlamydia trachomatis. Curr Opin Infect Dis. 2011;24(1):62-9. https://doi. org/10.1097/QCO.ob013e32834204d5 PMID: 21157332

License, supplementary material and copyright

This is an open-access article distributed under the terms of the Creative Commons Attribution (CC BY 4.0) Licence. You may share and adapt the material, but must give appropriate credit to the source, provide a link to the licence and indicate if changes were made.

Any supplementary material referenced in the article can be found in the online version.

This article is copyright of the authors or their affiliated institutions, 2019. 